\title{
Severe atypical pneumonia in critically ill patients: a retrospective multicenter study
}

CrossMark

S. Valade ${ }^{1,2^{*}}$, L. Biard ${ }^{2,3}$, V. Lemiale ${ }^{1,2}$, L. Argaud ${ }^{4}$, F. Pène ${ }^{5}$, L. Papazian ${ }^{6}$, F. Bruneel $^{7}$, A. Seguin ${ }^{8}$, A. Kouatchet ${ }^{9}$, J. Oziel ${ }^{10}$, S. Rouleau ${ }^{11}$, N. Bele ${ }^{12}$, K. Razazi $^{13}$, O. Lesieur ${ }^{14}$, F. Boissier ${ }^{15}$, B. Megarbane $^{16}$, N. Bigé ${ }^{17}$, N. Brulé ${ }^{18}$, A. S. Moreau ${ }^{19}$, A. Lautrette ${ }^{20}$, O. Peyrony ${ }^{21}$, P. Perez ${ }^{22}$, J. Mayaux ${ }^{23}$ and E. Azoulay ${ }^{1,2}$

\begin{abstract}
Background: Chlamydophila pneumoniae (CP) and Mycoplasma pneumoniae (MP) patients could require intensive care unit (ICU) admission for acute respiratory failure.

Methods: Adults admitted between 2000 and 2015 to 20 French ICUs with proven atypical pneumonia were retrospectively described. Patients with MP were compared to Streptococcus pneumoniae (SP) pneumonia patients admitted to ICUs.

Results: A total of 104 patients were included, 71 men and 33 women, with a median age of 56 [44-67] years. MP was the causative agent for 76 (73\%) patients and CP for 28 (27\%) patients. Co-infection was documented for 18 patients (viruses for 8 [47\%] patients). Median number of involved quadrants on chest $X$-ray was 2 [1-4], with alveolar opacities $(n=61,75 \%)$, interstitial opacities $(n=32,40 \%)$. Extra-pulmonary manifestations were present in 34 (33\%) patients. Mechanical ventilation was required for 75 (72\%) patients and vasopressors for 41 (39\%) patients. ICU length of stay was 16.5 [9.5-30.5] days, and 11 (11\%) patients died in the ICU. Compared with SP patients, MP patients had more extensive interstitial pneumonia, fewer pleural effusion, and a lower mortality rate [6 (8\%) vs. 17 (22\%), $p=0.013]$. According MCA analysis, some characteristics at admission could discriminate MP and SP. MP was more often associated with hemolytic anemia, abdominal manifestations, and extensive chest radiograph abnormalities. SP-P was associated with shock, confusion, focal crackles, and focal consolidation.
\end{abstract}

Conclusion: In this descriptive study of atypical bacterial pneumonia requiring ICU admission, mortality was $11 \%$. The comparison with SP pneumonia identified clinical, laboratory, and radiographic features that may suggest MP or CP pneumonia.

Keywords: Pneumonia, Outcome, ICU, Mycoplasma pneumoniae, Chlamydophila pneumoniae

\section{Background}

Severe pneumonia remains the major reasons for admission to the intensive care unit (ICU), mainly related to Streptococcus pneumoniae (SP). Atypical pneumonia (AP) related, for instance, to Chlamydophila pneumoniae (CP) and Mycoplasma pneumoniae (MP) accounts for $1-30 \%$ of documented pneumonia in patients admitted to ICU [1-11]. Although AP is rarely severe, some

*Correspondence: sandrine.valade@aphp.fr

1 AP-HP, Medical ICU, Hôpital Saint-Louis, 1 Avenue Claude Vellefaux, 75010 Paris, France

Full list of author information is available at the end of the article patients with community-acquired AP require ICU admission. Several retrospective studies reported ICU admission for $2-16.3 \%$ of patients with AP $[1-3,8,11-$ 15]. In one study, even $38.8 \%$ of patients with AP, older than 65 years, were admitted to ICU [12]. Among ICUadmitted patients with AP, $0.3-11 \%$ required mechanical ventilation $[4,5]$, with acute respiratory distress syndrome (ARDS) for few patients $[15,16]$. In previous studies, mortality rates were low, around $3 \%[5,13,14,17]$, although a recent retrospective study found $29.4 \%$ mortality [12] in a population with high rates of co-infection and cardiac complications. 
In previous non-ICU studies, compared to bacterial pneumonia, AP was associated with younger age and fewer comorbidities, a lower risk of severe respiratory failure, and better outcome $[4,6,13,14,18]$. For patients admitted to ICU, studies remained rare.

The main objective of the study was to describe AP in patients admitted to ICU. Our secondary objective was to compare the diagnostic strategy and outcomes between Mycoplasma pneumoniae-related pneumonia (MP) and Streptococcus pneumoniae-related pneumonia (SP) admitted to ICU.

\section{Methods}

\section{Patients with atypical pneumonia (AP)}

This is a retrospective chart review of adults admitted to 20 ICUs in France with a diagnosis of AP over the 16-year period from 2000 to 2015 (Additional file 1: Figure S1). Inclusion criteria were pneumonia defined with sepsis and a new pulmonary infiltrate on the chest radiograph and either a positive specific polymerase chain reaction (PCR) test for MP or CP on respiratory specimens (noninvasive samples or bronchoalveolar lavage) or blood serologic tests suggesting acute MP or CP infection (elevated specific IgM or fourfold increase in IgG level between two time points or elevated anti-MP IgG combined with presence of cold agglutinins) [19].

This study was approved by a local ethic committee (Société de Réanimation de Langue Française, CE SRLF 18-01).

\section{Data collection}

Clinical and laboratory data at ICU admission were collected, as well as organ failure during ICU stay. The SAPS II score [20] was used to assess severity at ICU admission. We also collected extra-pulmonary symptoms; arthritis was defined as new inflammation with one or more joints, myocarditis with cardiac dysfunction and troponin elevation and cutaneous involvement with the onset of skin rash. Bacterial and/or viral co-infections at diagnosis were recorded.

Patients with Streptococcus pneumoniae pneumonia (SP-P) Patients with MP-AP were compared to a group of consecutive patients with proven SP-P admitted to one of the study ICUs (Saint Louis Hospital, Paris) during the same period. SP-P was diagnosed based on sepsis with a new pulmonary infiltrate and identification of SP in at least one microbiological specimen (blood culture, respiratory specimen, or urinary antigen with no alternative diagnosis).

\section{Statistical analysis}

Categorical variables were described as $n$ (\%) and quantitative variables as median [25th-75th percentiles]. We first described the features in the patients with AP at ICU admission. Then, we conducted univariate analyses with a nonparametric test to compare the groups with MP-AP and SP-P. Finally, multiple correspondence analysis (MCA) was performed to identify the dimensions associated with the parameters at ICU admission (HIV, symptom duration, shock, confusion, diarrhea, physical chest findings, chest radiograph abnormalities, bilirubin level, and hemolytic anemia) and the causative organism, using the FactoMineR library in the R software platform. MCA is an extension of simple correspondence analysis designed to analyze relations among categorical variables. The aim is to redefine the principal dimensions or axes of the space in a way that captures the highest possible percentage of the inertia (which can be likened to the explained variance).

All tests were two-tailed. $p$ values $<0.05$ were considered significant. All statistical analyses were carried out using the R 2.13.1 statistical platform (http://www.Rproject.org).

\section{Results \\ Clinical findings in the patients with atypical pneumonia (AP)}

We included 104 patients, 71 men and 33 women, with a median age of 56 [44-67] years (Additional file 2: Table S2). Acute respiratory failure was the main reason for ICU admission $(n=96$; $92 \%)$; other reasons were cardiovascular failure $(n=2)$, neurological disorders $(n=3)$, and miscellaneous reasons $(n=3)$.

AP was more common in the fall and winter (Fig. 1). Furthermore, AP became more common over time, suggesting improved diagnosis after the introduction of PCR testing.

Table 1 and Additional file 3: Table S1 report the main features of the patients with AP. The most common comorbidity was chronic respiratory disease, which was present in 32 (31\%) patients including 9 patients with chronic obstructive lung disease, 4 patients with asthma, and 4 patients with interstitial lung disease; of these 32 patients, 7 patients were on long-term oxygen therapy before ICU admission. Immunosuppression was noted in 21 patients including $10(48 \%)$ with hematological malignancies (lymphoma, $n=6$ ), 7 with solid cancer, and 2 with HIV infection. Delay from respiratory symptoms onset to ICU admission was 5 [3-8] days. A fever defined with a body temperature above $38.5^{\circ} \mathrm{C}$ was present in 77 patients (74\%). At ICU admission, all patients were tachypneic (respiratory rate, $32[26-37] / \mathrm{min}$ ) and 


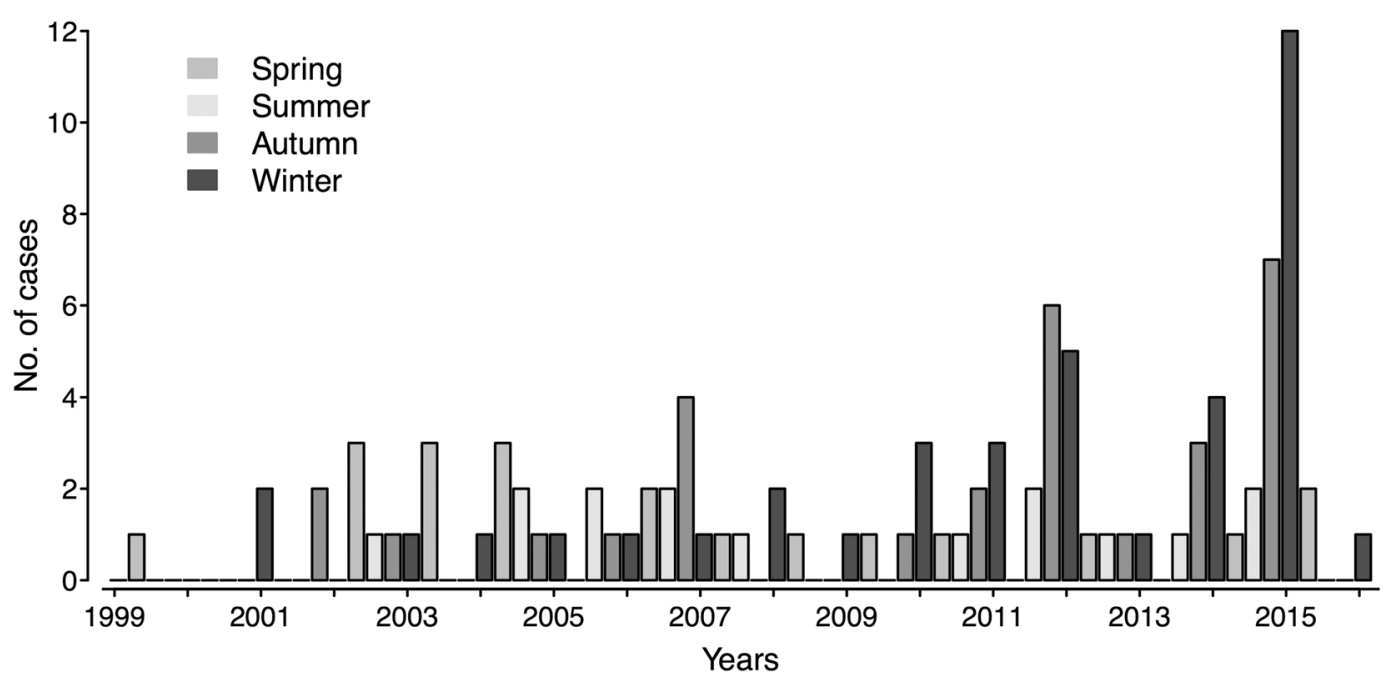

Fig. 1 Distribution of atypical pneumonia cases by season and year

48 (46\%) had severe respiratory symptoms. Physical chest examination included crackles $(n=54 ; 52 \%)$, rhonchi ( $n=15 ; 14 \%)$, wheezing $(n=12 ; 11 \%)$, and signs of consolidation $(n=7 ; 7 \%)$. No squeaks were reported. Extrapulmonary symptoms concerned 34 (33\%) patients and included arthritis $(n=2)$, myocarditis $(n=4)$, and skin rash $(n=6)$. Almost one-third of the patients $(n=32$; $31 \%)$ had neurological symptoms at ICU admission, mostly with an altered level of consciousness related to severity of sepsis or to hypoxemia. Confusion was the main symptom for $3(3 \%)$ patients, and meningoencephalitis was diagnosed in 1 patient. Cold agglutinins assessed in case of hemolytic anemia were positive in 9 (9\%) MP patients, cytolysis occurred in $11(10 \%)$ patients, and rhabdomyolysis was present in $3(3 \%)$ patients. At ICU admission, 10 (10\%) patients had shock, the SOFA score was 5 [2-7], and the SAPS II was 33 [25-44].

\section{Other findings in patients with atypical pneumonia (AP)}

The most common findings by chest radiography were alveolar opacities $(n=61,59 \%)$, and interstitial opacities $(n=32,31 \%)$ in $2[1-4]$ quadrants. Pleural effusion was rare $(n=6,6 \%)$.

The causative organism was MP in 76 (73\%) patients and CP in 28 (27\%) patients and was identified by serological testing (positive IgM or elevated IgG) in 71 patients, positive PCR on respiratory samples in 33 patients (18 on bronchoalveolar lavage, 10 on naso-pharyngeal aspirate, 2 on tracheal aspirate and 4 on nasal swab) and by both diagnostic methods in 5 patients. None of the collected variables differed between patients diagnosed with PCR, serology or both (Additional file 4: Table S3). Co-infection was found in $18(20 \%)$ patients and was related to viruses ( $n=9$; influenza, rhinovirus, respiratory syncytial virus, coronavirus) or bacteria ( $n=6$; Haemophilus influenzae, Proteus mirabilis, Staphylococcus aureus, Serratia marcescens) or Pneumocystis jirovecii $(n=3)$. None of MP patients had co-infection with CP or SP.

\section{ICU management of atypical pneumonia (AP)}

Mechanical ventilation was required for 75 (72\%) patients and lasted 13 [8-19] days. Of the 34 (45\%) patients meeting criteria for ARDS, 4 required extracorporeal membrane oxygenation. Vasoactive agents were required for $41(39 \%)$ patients, and renal replacement therapy was started for $10(10 \%)$ patients.

The first-line antibiotics were active on MP and $\mathrm{CP}$ in $62(60 \%)$ patients. Time from ICU admission to antibiotic initiation was 1 [0-4] day. Combination therapy was used in 61 (59\%) patients and consisted to a third-generation cephalosporin (C3G) and a macrolide in 24 (39\%) patients, a C3G and a quinolone in 13 (21\%) patients, another betalactam and a macrolide in $16(26 \%)$ patients, another betalactam and a quinolone in $6(9 \%)$ patients, or another antibiotic and a macrolide in $2(3 \%)$ patients. Antibiotics was adapted according to microbiology results with a macrolide $(n=72)$, a quinolone $(n=24)$ or a cycline $(n=3)$.

\section{Outcomes of atypical pneumonia (AP)}

Eleven (11\%) patients died in the ICU. ICU stay length was 16.5 [9.5-30.5] days. Persistent hypoxemia was present at ICU discharge in 60 (58\%) patients. By univariate analysis, factors associated with mortality were age $\geq 65$ years $(p=0.033)$, signs of respiratory distress $(p=0.017)$, and interstitial opacities on the chest 
Table 1 Clinical characteristics of patients with atypical pneumonia at ICU admission and outcome according to the causative agent

\begin{tabular}{|c|c|c|c|}
\hline$N(\%)$ or median [IQR] & Total $(N=104)$ & Mycoplasma pneumoniae $(N=76)$ & $\begin{array}{l}\text { Chlamydophila } \\
\text { pneumoniae } \\
(N=28)\end{array}$ \\
\hline \multicolumn{4}{|l|}{ Demographics } \\
\hline Age & $56[44-67]$ & $54[41-69]$ & $64[52-75]$ \\
\hline Female gender & $33(32 \%)$ & $26(34 \%)$ & $7(25 \%)$ \\
\hline \multicolumn{4}{|l|}{ Comorbidities } \\
\hline Chronic respiratory disease & $32(31 \%)$ & $22(29 \%)$ & $10(36 \%)$ \\
\hline Current smoker & $30(29 \%)$ & $20(38 \%)$ & $10(36 \%)$ \\
\hline Immunosuppression & $21(20 \%)$ & $17(22 \%)$ & $4(14 \%)$ \\
\hline HIV infection & $2(2 \%)$ & $2(3 \%)$ & 0 \\
\hline Hematological malignancy & $10(10 \%)$ & $9(12 \%)$ & $1(3.5 \%)$ \\
\hline Cancer & $7(7 \%)$ & $4(5 \%)$ & $3(11 \%)$ \\
\hline Hypertension & $32(31 \%)$ & $24(32 \%)$ & $8(28 \%)$ \\
\hline \multicolumn{4}{|l|}{ Reason for ICU admission } \\
\hline Acute respiratory distress & $96(92 \%)$ & $70(92 \%)$ & $26(93 \%)$ \\
\hline Cardiovascular failure & $2(2 \%)$ & $2(3 \%)$ & 0 \\
\hline Neurological disorders & $33 \%)$ & $2(3 \%)$ & $1(3.6 \%)$ \\
\hline Other & $3(3 \%)$ & $2(3 \%)$ & $1(3.6 \%)$ \\
\hline \multicolumn{4}{|l|}{ Clinical respiratory findings } \\
\hline Respiratory rate & $32[26-37]$ & 33 [27-38] & 30 [26-33] \\
\hline Signs of respiratory failure & $48(46 \%)$ & $33(49 \%)$ & $15(54 \%)$ \\
\hline Rhonchi & $15(14 \%)$ & $9(15 \%)$ & $6(21 \%)$ \\
\hline Crackles & $54(52 \%)$ & $36(47 \%)$ & $18(64 \%)$ \\
\hline Signs of consolidation & $7(7 \%)$ & $5(9 \%)$ & $2(7 \%)$ \\
\hline Decreased vesicular breath sounds & $14(13 \%)$ & $10(17 \%)$ & $4(14 \%)$ \\
\hline \multicolumn{4}{|l|}{ Clinical presentation } \\
\hline Time since symptom onset (days) & $5[3-8]$ & $6[4-9]$ & $4[2-7]$ \\
\hline Fever & $77(74 \%)$ & $58(83 \%)$ & $19(68 \%)$ \\
\hline Shock & $10(10 \%)$ & $6(8 \%)$ & $4(14 \%)$ \\
\hline Neurological symptoms & $32(31 \%)$ & $19(25 \%)$ & $13(46 \%)$ \\
\hline Gastrointestinal symptoms & $1(1 \%)$ & $1(1 \%)$ & 0 \\
\hline \multicolumn{4}{|l|}{ Extra-pulmonary signs } \\
\hline$\geq 1$ extra-pulmonary symptom & $34(33 \%)$ & $27(36 \%)$ & $7(25 \%)$ \\
\hline Arthritis & $2(2 \%)$ & $1(1 \%)$ & $1(3.5 \%)$ \\
\hline Myocarditis & $4(4 \%)$ & $4(5 \%)$ & 0 \\
\hline \multicolumn{4}{|l|}{ Treatments in the ICU } \\
\hline Mechanical ventilation & $75(72 \%)$ & $50(66 \%)$ & $25(89 \%)$ \\
\hline Duration of ventilation & $13[8-19]$ & $12.5[8-22.5]$ & $13.5[8.5-19]$ \\
\hline Vasopressors & $41(39 \%)$ & $26(34 \%)$ & $15(54 \%)$ \\
\hline Renal replacement therapy & $10(9.5 \%)$ & $7(9 \%)$ & $3(11 \%)$ \\
\hline \multicolumn{4}{|l|}{ Outcomes } \\
\hline Death in the ICU & $11(10 \%)$ & $6(8 \%)$ & $5(18 \%)$ \\
\hline \multicolumn{4}{|l|}{ Length of ICU stay (days) } \\
\hline Discharged alive & $15[8-26]$ & $15[8-27]$ & $19[12-24]$ \\
\hline ICU death & 39 [25-49] & $37[26-47]$ & 39 [25-90] \\
\hline
\end{tabular}

HIV human immunodeficiency virus, ICU intensive care unit 
radiograph $(p=0.017)$. For MP patients, $26(34 \%)$ did not receive adequate antibiotic at ICU admission. Among them 2 patients died.

\section{Comparison to Streptococcus pneumoniae pneumonia (SP-P)}

Tables 2 and 3 reports univariate analysis comparing patients with MP-AP and SP-P. Factors significantly associated with SP-P were HIV infection [12 (16\%) vs. $2(3 \%)$, $p=0.009$, neurological symptoms [20 (26\%) vs. $1(1 \%)$, $p<0.0001]$, and gastrointestinal symptoms [15 (20\%) vs. $1(1 \%), p=0.0003]$. Factors significantly associated with MP were hemolytic anemia or cold agglutinins [0 (0\%) vs. $9(12 \%), p=0.003]$. Also, 6 patients with SP-P had coinfection (influenza A, $n=3$; Haemophilus influenzae, $n=2$; Streptococcus constellatus, $n=1$ ).

SP-P was associated with a shorter length of respiratory symptoms before ICU admission (3 days [2-7] vs. 6 days [4-9], $p=0.0008)$. At ICU admission SAPS II score was higher for SP-P (42 [30-55] vs. 32 [22-41], $p=0.005)$, shock was more frequent $(32 \%$ vs. $8 \% ; p=0.0004)$, creatinine level was higher (101 [69.5-168.8] $\mu \mathrm{mol} / \mathrm{L}$ vs. 77 [57.5-108] $\mu \mathrm{mol} / \mathrm{L}, p=0.008)$, and lactate level was high $(2.3[1.8-3.4] \mathrm{mmol} / \mathrm{l}$ vs. $1[0.07-2.7] \mathrm{mmol} / \mathrm{l} ; p=0.003)$.

Signs of consolidation and decreased breath sounds were more common in SP-P than in MP-AP (30\% vs. $9 \%$ and $38 \%$ vs. $17 \%$, respectively). MP-AP involved 4 quadrants on chest X-ray ( $26 \%$ vs. $8 \%, p=0.013)$ but less frequently pleural space ( $5 \%$ vs. $11 \%, p=0.007$ ). The bilirubin level was higher in the patients with SP-P (15 [9.2-24.5] $\mu \mathrm{mol} / \mathrm{L}$ vs. 8.4 [5.8-13] $\mu \mathrm{mol} / \mathrm{L}, p=0.0006)$. MP-AP was associated with the use of mechanical ventilation $(66 \%$ vs. $50 \%, p=0.049)$. ICU length of stay (LOS) seemed prolonged in case of MP-AP regardless of the ICU outcome (median LOS 37 vs. 5 days and 15 vs. 5 days, respectively, in patients who died in the ICU and in patients who were discharged alive). However, 28-day mortality was lower in the MP-AP group (5\% vs. $20 \%$, $p=0.005)$.

Figure 2 shows the MCA results for the clinical and radiological characteristics at admission. Several characteristics discriminated between MP and CP. MP was more often associated with hemolytic anemia, abdominal manifestations and extensive chest radiograph abnormalities. SP-P was associated with shock, confusion, focal crackles, and focal consolidation.

\section{Discussion}

This multicenter study is the largest one analyzing 104 AP patients admitted to ICU. Extra-pulmonary symptoms were seen for one-third of patients, corresponding to data on previous study for patients not admitted to ICU [21]. However, AP in non-ICU patients was described as mild [6], whereas a substantial proportion of our patients had severe acute pneumonia, with shock at ICU admission for $10 \%$ of patients and mechanical ventilation required for $72 \%$ of patients including $45 \%$ of patients with ARDS.

In previous studies, patients with MP-AP were younger and had fewer comorbidities, lower respiratory disease severity and better outcomes $[4,6,13,14,18]$. In our study, with ICU patients, age was similar for patients with MP-AP and SP-P.

Previous studies also compared clinical and radiological features according to the causative organism of pneumonia $[8,15,18]$. In a Japanese cohort, among patients with pneumonia and audible crackles, these were more often heard only in late inspiration in patients with AP and throughout inspiration in patients with other bacteria [22]. In our study, patients with MP-AP had no specific clinical findings, except signs of consolidation which were associated with SP-P. On radiological findings, compared to SP-P, MP-AP was more often responsible for ground-glass opacification, centrilobular nodules, bronchial wall thickening, and diffuse radiological abnormalities [1, 15, 18]. In our study, extensive interstitial pneumonia was more common in MP-AP than in SP-P.

The Japanese Respiratory Society published guidelines for identifying MP-AP [17] and established a scoring system based on six parameters: age $<60$ years, minor or no comorbidities, stubborn cough, abnormal chest auscultation, the absence of sputum and of an etiological agent identifiable by rapid diagnostic testing, and peripheral white blood cell count $<10,000 / \mu \mathrm{L}$. A score $\geq 4$ indicates a high probability of MP-AP (sensitivity, 88.7\%; and specificity, 77.5\%). Another scoring system performed well in separating patients with pneumonia into three groups: pyogenic bacteria; MP, CP, or virus; and unknown agent [23]. Nevertheless, neither scoring system had been assessed in ICU patients. In our study, MCA provided insights into differences between MP-AP and SP-P. Hemolytic anemia, diffuse chest radiograph abnormalities, and interstitial opacities were associated with MP-AP. On the contrary, HIV infection, shock, neurological symptoms, gastrointestinal symptoms, signs of consolidation, shorter symptom duration, higher bilirubin level, and radiological alveolar opacities were strongly linked to SP-P.

Compared to patients with SP-P, those with MP-AP more often required mechanical ventilation and spent more time in the ICU yet had a lower risk of death. This lower mortality may be ascribable to the smaller number of MP-AP patients with extra-pulmonary organ failure (shock, neurological manifestations, acute renal failure) and to the lower SAPS II severity score in the MP-AP group (32 [22-41] vs. 42 [30-55], $p=0.005$ ). 
Table 2 Univariate analysis comparing clinical characteristics and outcomes of patients with Mycoplasma pneumoniae versus Streptococcus pneumoniae pneumonia

\begin{tabular}{|c|c|c|c|c|}
\hline$N(\%)$ or median (IQR) & Total $(N=152)$ & $\begin{array}{l}\text { Mycoplasma pneumoniae } \\
(N=76)\end{array}$ & $\begin{array}{l}\text { Streptococcus pneumoniae } \\
(N=76)\end{array}$ & $p$ value \\
\hline \multicolumn{5}{|l|}{ Demographics } \\
\hline Age & 55 [43-69] & 54 [41-69] & $57[44-73]$ & 0.058 \\
\hline Female gender & $51(34 \%)$ & $26(34 \%)$ & $25(33 \%)$ & 1 \\
\hline \multicolumn{5}{|l|}{ Comorbidities } \\
\hline Chronic respiratory disease & $36(24 \%)$ & $22(29 \%)$ & $14(18 \%)$ & 0.18 \\
\hline Current smoker & $49(41 \%)$ & $20(38 \%)$ & $29(43 \%)$ & \\
\hline Immunosuppression & $44(29 \%)$ & $17(22 \%)$ & $27(36 \%)$ & 0.11 \\
\hline HIV infection & $14(9 \%)$ & $2(3 \%)$ & $12(16 \%)$ & 0.009 \\
\hline Hematological malignancy & $18(12 \%)$ & $9(12 \%)$ & $9(12 \%)$ & 1 \\
\hline Cancer & $12(8 \%)$ & $4(5 \%)$ & $8(11 \%)$ & 0.37 \\
\hline Hypertension & $50(33 \%)$ & $24(32 \%)$ & $26(34 \%)$ & 0.86 \\
\hline \multicolumn{5}{|l|}{ Reason for ICU admission } \\
\hline Acute respiratory distress & $140(92 \%)$ & $70(92 \%)$ & 70 (92\%) & 0.59 \\
\hline Shock & $6(4 \%)$ & $2(3 \%)$ & $4(5 \%)$ & \\
\hline Neurological symptoms & $4(3 \%)$ & $2(3 \%)$ & $2(3 \%)$ & \\
\hline Other & $2(1 \%)$ & $2(3 \%)$ & 0 & \\
\hline \multicolumn{5}{|l|}{ Clinical respiratory findings } \\
\hline Respiratory rate & 31 [26-38] & 33 [27-38] & 30 [26-36] & 0.43 \\
\hline Signs of respiratory distress & $67(47 \%)$ & $33(49 \%)$ & $34(45 \%)$ & 0.74 \\
\hline Rhonchi & $21(16 \%)$ & $9(15 \%)$ & $12(16 \%)$ & 1 \\
\hline Crackles & $79(59 \%)$ & $36(61 \%)$ & $44(59 \%)$ & 1 \\
\hline Signs of consolidation & $27(21 \%)$ & $5(9 \%)$ & $22(30 \%)$ & 0.008 \\
\hline Decreased vesicular breath sounds & $38(28 \%)$ & $10(17 \%)$ & $28(38 \%)$ & 0.007 \\
\hline \multicolumn{5}{|l|}{ Clinical presentation } \\
\hline Time since symptom onset (days) & $4[2-7]$ & $6[4-9]$ & $3[2-7]$ & 0.0008 \\
\hline Fever & $112(77 \%)$ & $58(83 \%)$ & $54(71 \%)$ & 0.12 \\
\hline Shock & $30(20 \%)$ & $6(8 \%)$ & $24(32 \%)$ & 0.0004 \\
\hline Neurological symptoms & $21(14 \%)$ & $1(1 \%)$ & $20(26 \%)$ & $<0.0001$ \\
\hline Gastrointestinal symptoms & $16(11 \%)$ & $1(1 \%)$ & $15(20 \%)$ & 0.0003 \\
\hline \multicolumn{5}{|l|}{ Extra-pulmonary signs } \\
\hline$\geq 1$ extra-pulmonary sign & $66(43 \%)$ & $27(36 \%)$ & $39(51 \%)$ & 0.071 \\
\hline Arthritis & $1(1 \%)$ & $1(1 \%)$ & 0 & 1 \\
\hline Myocarditis & $4(3 \%)$ & $4(5 \%)$ & 0 & 0.12 \\
\hline \multicolumn{5}{|l|}{ Treatments in the ICU } \\
\hline Mechanical ventilation & $88(58 \%)$ & $50(66 \%)$ & $38(50 \%)$ & 0.049 \\
\hline \multicolumn{5}{|l|}{ Duration of ventilation (days) } \\
\hline Discharged alive & $11[7-19]$ & 13 [8-23] & $9[6-16]$ & \\
\hline ICU death & $11[3-18]$ & 18 [17-34] & $5[2-15]$ & \\
\hline Vasopressors & $60(39 \%)$ & $26(34 \%)$ & $34(45 \%)$ & 0.26 \\
\hline Renal replacement therapy & $17(11 \%)$ & $7(9 \%)$ & $10(13 \%)$ & 0.49 \\
\hline SAPS $\|$ & 36 [24-47] & $32[22-41]$ & $42[30-55]$ & 0.0005 \\
\hline \multicolumn{5}{|l|}{ Outcomes } \\
\hline \multicolumn{5}{|l|}{ ICU stay length (days) } \\
\hline Discharged alive & $9[5-19]$ & 15 [8-27] & $5[3-10]$ & \\
\hline ICU death & 13 [4-27] & $37[26-47]$ & $5[3-14]$ & \\
\hline 28-day mortality & $23(15 \%)$ & $6(8 \%)$ & $17(22 \%)$ & 0.013 \\
\hline
\end{tabular}

HIV human immunodeficiency virus, ICU intensive care unit, SAPS II Simplified Acute Physiology Score version II 
Table 3 Univariate analysis comparing laboratory findings in patients with Mycoplasma pneumoniae versus Streptococcus pneumoniae pneumonia

\begin{tabular}{|c|c|c|c|c|}
\hline$N(\%)$ or median (IQR) & Total $(N=152)$ & $\begin{array}{l}\text { Mycoplasma pneumoniae } \\
\text { patients }(N=76)\end{array}$ & $\begin{array}{l}\text { Streptococcus pneumoniae } \\
\text { patients }(N=76)\end{array}$ & $p$ value \\
\hline \multicolumn{5}{|l|}{ Laboratory features } \\
\hline Lactate $(\mathrm{mmol} / \mathrm{l})$ & $2.2[1.6-3.3]$ & $1[0.7-2.7]$ & $2.3[1.8-3.4]$ & 0.003 \\
\hline $\mathrm{P} /$ F ratio & 163 [92-267] & $120[88-236]$ & $178[114-280]$ & 0.051 \\
\hline Serum sodium (mmol/L) & 136 [133-139] & $137[135-140]$ & 136 [132-139] & 0.028 \\
\hline Creatinine $(\mu \mathrm{mol} / \mathrm{L})$ & 87 [65-139.5] & $77[57.5-108]$ & $101[69.5-168.8]$ & 0.008 \\
\hline CPK (IU/I) & 122 [40-309] & 138 [89-608] & $108[36-202]$ & 0.093 \\
\hline ASAT (IU/I) & 38 [23-80] & $44[24-81]$ & 38 [22-77] & 0.45 \\
\hline Bilirubin $(\mu \mathrm{mol} / \mathrm{l})$ & $12.8[8-21.7]$ & $8.4[5.8-13]$ & $15[9.2-24.5]$ & 0.0006 \\
\hline Leukocytes & $11,400[7200-16,300]$ & $11,140[8100-17,000]$ & $11,200[5112-16,142]$ & 0.63 \\
\hline Hemoglobin (g/dL) & $11.6[10-12.9]$ & $11.3[9.6-13.1]$ & $11.6[10.2-12.8]$ & 0.89 \\
\hline Platelets (Giga/L) & 217 [138-287] & $262.5[179.5-311.25]$ & 204 [138-252] & 0.009 \\
\hline Cytolysis & $21(14 \%)$ & $8(11 \%)$ & $13(17 \%)$ & 0.35 \\
\hline Hemolytic anemia/cold agglutinins & $9(6 \%)$ & $9(12 \%)$ & 0 & 0.003 \\
\hline Rhabdomyolysis & $5(3 \%)$ & $2(3 \%)$ & $3(4 \%)$ & 1 \\
\hline \multicolumn{5}{|l|}{ Radiological features } \\
\hline Number of quadrants involved & & & & 0.013 \\
\hline$\leq 2$ & $103(68 \%)$ & $37(49 \%)$ & $66(87 \%)$ & \\
\hline$>2$ & $25(16 \%)$ & $16(21 \%)$ & $9(12 \%)$ & \\
\hline Alveolar opacities & $111(85 \%)$ & $42(75 \%)$ & $19(68 \%)$ & 0.013 \\
\hline Interstitial opacities & $26(20 \%)$ & $20(36 \%)$ & $12(43 \%)$ & 0.0001 \\
\hline Pleural effusion & $20(15 \%)$ & $3(5 \%)$ & $3(11 \%)$ & 0.007 \\
\hline
\end{tabular}

P/F ratio ratio of partial pressure of oxygen in arterial blood over fraction of inspired oxygen, CPK creatine phosphokinase, ASAT aspartate aminotransferase

Interestingly, intracellular pathogens are underdiagnosed like viruses, but under-covered despite the availability of therapeutic agents. These findings are in line with these from Menendez et al. [24] who reported a lack of antibiotic compliance in patients with CAP. Our descriptive data may be useful to help clinicians to discriminate SP-related pneumonia and MP-related pneumonia, even if a double antibiotherapy active against atypical pathogens is recommended in severe patients.

This study had several limitations. First, the study design was retrospective and patients were included within a 16-year period. ICU management may have changed over this period. ICU admissions criteria could be different according to the center and the year of admission. Atypical pneumonia remains rare, and the main objective of the study was to describe AP in the most severe patients. However, the study assessed mostly the clinical and radiological characteristics at admission which would be unlikely to change between the centers.

Secondarily, SP-P patients were included from only one single center, whereas AP patients were included from several centers. The main objective of the study was to describe patients at ICU admission. Although admission rules would be different between the centers, the clinical presentation would not be affected. Thirdly, only patients with proven AP based on positive microbiologic samples were included. Half of the patients with MP-AP had their diagnosis based on serological testing. More recently only PCR was used to diagnose Mycoplasma pneumoniae infection. Positivity of IgM anti-MP is considered as the gold standard, and PCR sensitivity is equal [25]. Although some of the patients had serological tests with fourfold increase in IgG level between two time points, we believe that we included only proven MP-AP patients. Although different diagnostic tests were used within the study period and among the centers, those tests were enough sensitive and specific to include real MP-AP pneumonia.

Fourth, we did not include patients with Legionella pneumophila pneumonia, a more frequent atypical pneumonia. Although Legionella pneumophila pneumonia was associated with higher risk of ICU admission comparing to MP-AP and CP-AP, our goal was to focus on AP that is usually non-severe and only occasionally leads to ICU admission. Moreover, several studies analyzed Legionella pneumophila pneumonia. Similarly, we did not include more rare etiology of pneumonia as $\mathrm{Q}$ fever. 


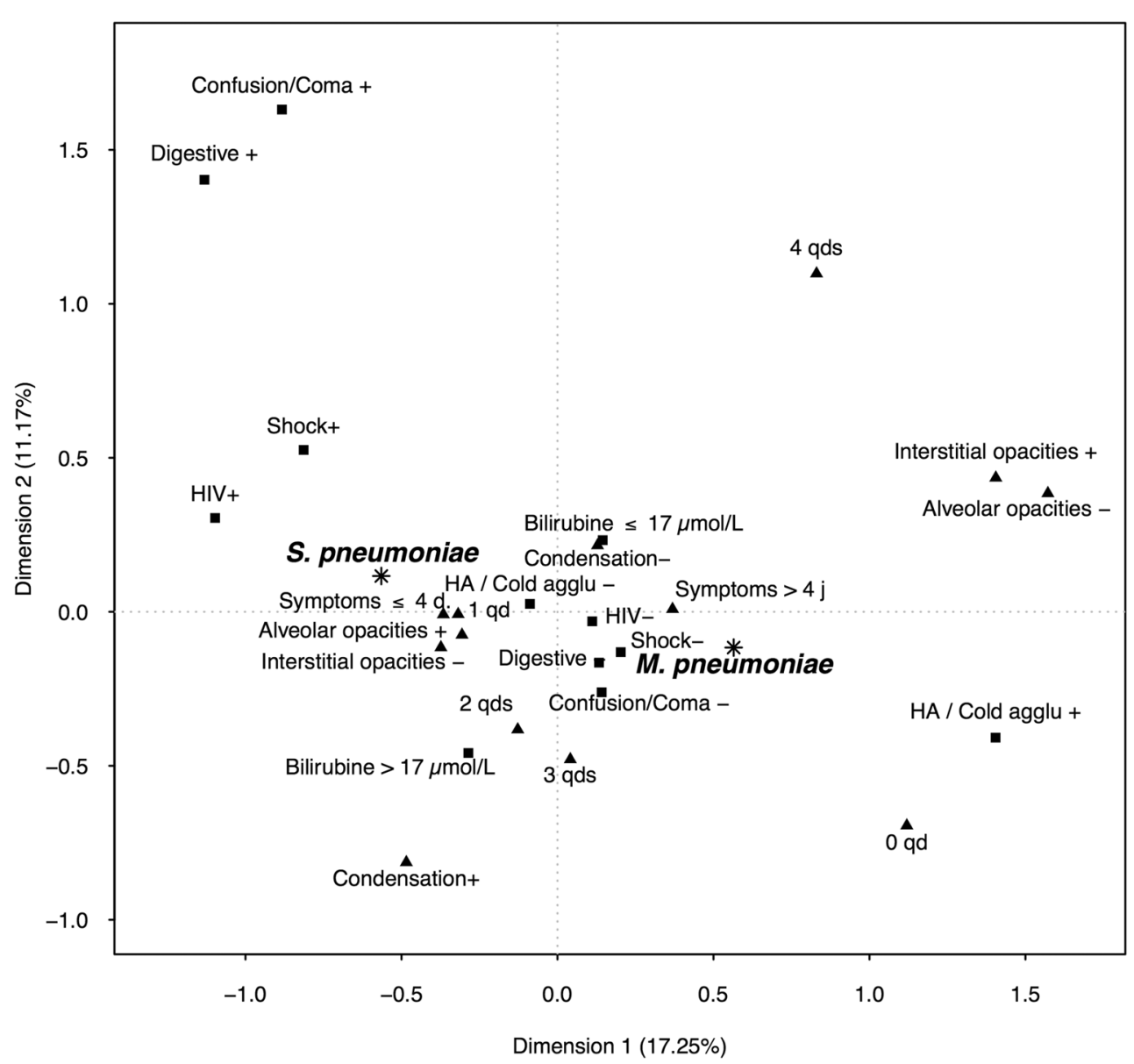

Fig. 2 Multiple correspondence analysis: the factors are mapped along two dimensions. Triangles indicate pulmonary signs and squares extra-pulmonary signs. HA hemolytic anemia, Cold agglu+ presence of cold agglutinins, QD quadrants

\section{Conclusion}

Although considered as less severe pneumonia, atypical pneumonia requiring ICU admission remained associated with $11 \%$ mortality. At ICU admission, several clinical and radiological features could differ between MP-AP and SP-P, which may help physicians. Prospective studies are needed to validate clinical model to AP in ICU patients.

\section{Additional files}

Additional file 1: Figure S1. Flow chart: ICU admission and diagnostic strategy.

Additional file 2: Table S2. Cases distribution (MP and CP-AP patients) by center.

Additional file 3: Table S1. Laboratory findings in patients with atypical pneumonia according to the causative agent.

Additional file 4: Table S3. Clinical characteristics of patients with atypical pneumonia at ICU admission and outcome according to the diagnostic methods.

\section{Abbreviations}

AP: atypical pneumonia; ARDS: adult respiratory distress syndrome; CP: Chlamydophila pneumoniae; ICU: intensive care unit; MCA: multiple correspondence analysis; MP: Mycoplasma pneumoniae; MP-AP: atypical pneumonia due to Mycoplasma pneumoniae; PCR: polymerase chain reaction; SAPS II: Simplified Acute Physiology Score version II; SP: Streptococcus pneumoniae; SP-P: pneumonia due to Streptococcus pneumoniae.

\section{Authors' contributions}

EA is the guarantor for the content of the manuscript, including the data and analysis. SV, LB, VL, and EA contributed substantially to the study design, data analysis and interpretation, and the writing of the manuscript. SV, VL, LA, FP, LP, $\mathrm{FB}, \mathrm{AS}, \mathrm{AK}, \mathrm{JO}, \mathrm{DS}, \mathrm{NB}, \mathrm{KR}, \mathrm{OL}, \mathrm{FB}, \mathrm{BM}, \mathrm{NB}, \mathrm{NB}, \mathrm{ASM}, \mathrm{AL}, \mathrm{OP}, \mathrm{PP}, \mathrm{JM}$, and EA contributed substantially to patients recruitment, collecting data, and manuscript revision. All authors read and approved the final manuscript.

\section{Author details}

${ }^{1}$ AP-HP, Medical ICU, Hôpital Saint-Louis, 1 Avenue Claude Vellefaux, 75010 Paris, France. ${ }^{2}$ UFR de Médecine, University Paris-7 Paris-Diderot, Paris, France. ${ }^{3}$ AP-HP, DBIM, Hôpital Saint-Louis, Paris, France. ${ }^{4}$ Hôpital Edouard Herriot, Service de Réanimation Médicale, Hospices Civils de Lyon, Lyon, France. ${ }^{5}$ AP-HP, Réanimation médicale, Hôpital Cochin, Paris, France. ${ }^{6}$ Réanimation des Détresses Respiratoires et Infections Sévères, Assistance Publique-Hôpitaux de Marseille, Hôpital Nord, Marseille, France. ${ }^{7}$ Service de Réanimation, Centre Hospitalier de Versailles, Le Chesnay, France. ${ }^{8}$ Department of Medical Intensive Care, CHU de Caen, Caen, France. ${ }^{9}$ Service de Réanimation Médicale et Médecine Hyperbare, Hôpital Angers, Angers, France. ${ }^{10}$ AP-HP, 
Medical-Surgical Intensive Care Unit, Avicenne University Hospital, Bobigny, France. ${ }^{11}$ Service de Réanimation polyvalente, Angoulême, France. ${ }^{12}$ Intensive Care Unit, Draguignan Hospital, Draguignan, France. ${ }^{13}$ AP-HP, Groupe Henri Mondor-Albert Chenevier, Service de Réanimation Médicale, Hôpital Henri Mondor, Créteil, France. ${ }^{14}$ Service de Réanimation, CH Saint-Louis, La Rochelle, France. ${ }^{15}$ AP-HP, Réanimation médicale, Hôpital Européen Georges Pompidou, Paris, France. ${ }^{16}$ AP-HP, Department of Medical and Toxicological Critical Care, Lariboisière Hospital, Paris, France. ${ }^{17}$ AP-HP, Medical Intensive Care Unit, Hôpital Saint-Antoine, Paris, France. ${ }^{18}$ Medical Intensive Care Unit, Centre Hospitalier Universitaire de Nantes, Nantes, France. ${ }^{19}$ Centre de réanimation, Hôpital Salengro, CHU-Lille, Lille, France. ${ }^{20}$ Service de Réanimation Médicale Polyvalente, CHU Gabriel Montpied, Clermont-Ferrand, France. ${ }^{21}$ AP-HP, Service des urgences, Hôpital Saint-Louis, Paris, France. ${ }^{22}$ Service de Réanimation médicale, Hôpital Brabois, Nancy, France. ${ }^{23}$ AP-HP, Pneumology and Critical Care Medicine Department, Universitary Hospital La Pitié Salpêtrière-Charles Foix, Paris, France.

\section{Acknowledgements}

None.

\section{Competing interests}

There is no financial or other competing interest in relation to this manuscript.

\section{Availability of data and materials}

The dataset supporting the conclusions of this article is included within the article (and its additional files).

\section{Consent for publication}

Not applicable.

\section{Ethics approval and consent to participate}

This study was approved by a local ethic committee (commission d'éthique de la Société de Réanimation de Langue Française, CE 18-01). At ICU admission, each patient and his family receive a leaflet informing them of the existence of clinical studies in the ward and the collection of data with the possibility to decline the use of these data.

\section{Funding}

No part of the work presented has received financial support from any source.

\section{Publisher's Note}

Springer Nature remains neutral with regard to jurisdictional claims in published maps and institutional affiliations.

Received: 12 April 2018 Accepted: 2 August 2018

Published online: 13 August 2018

\section{References}

1. Cillóniz C, Torres A, Niederman M, van der Eerden M, Chalmers J, Welte T, et al. Community-acquired pneumonia related to intracellular pathogens. Intensive Care Med. 2016:42(9):1374-86.

2. Ngeow Y-F, Suwanjutha S, Chantarojanasriri T, Wang F, Saniel M, Alejandria $\mathrm{M}$, et al. An Asian study on the prevalence of atypical respiratory pathogens in community-acquired pneumonia. Int J Infect Dis. 2005;9(3):144-53.

3. Saraya T, Kurai D, Nakagaki K, Sasaki Y, Niwa S, Tsukagoshi H, et al. Novel aspects on the pathogenesis of Mycoplasma pneumoniae pneumonia and therapeutic implications. Front Microbiol. 2014 Aug 11. Cited 2015 Jun 24; 5. http://www.ncbi.n/m.nih.gov/pmc/articles/PMC4127663/.

4. von Baum H, Welte T, Marre R, Suttorp N, Lück C, Ewig S. Mycoplasma pneumoniae pneumonia revisited within the German Competence Network for Community-acquired pneumonia (CAPNETZ). BMC Infect Dis. 2009;9:62

5. Marrie TJ. Mycoplasma pneumoniae pneumonia requiring hospitalization, with emphasis on infection in the elderly. Arch Intern Med. 1993:153(4):488-94.

6. Dumke R, Schnee C, Pletz MW, Rupp J, Jacobs E, Sachse K, et al. Mycoplasma pneumoniae and Chlamydia spp. infection in community-acquired pneumonia, Germany, 2011-2012. Emerg Infect Dis. 2015;21(3):426-34.

7. Spoorenberg SM, Bos WJW, Heijligenberg R, Voorn PG, Grutters JC, Rijkers GT, et al. Microbial aetiology, outcomes, and costs of hospitalisation for community-acquired pneumonia; an observational analysis. BMC Infect Dis. 2014;17(14):335.

8. Sohn JW, Park SC, Choi Y-H, Woo HJ, Cho YK, Lee JS, et al. Atypical pathogens as etiologic agents in hospitalized patients with communityacquired pneumonia in Korea: a prospective multi-center study. J Korean Med Sci. 2006;21(4):602-7.

9. Walden AP, Clarke GM, McKechnie S, Hutton P, Gordon AC, Rello J, et al. Patients with community acquired pneumonia admitted to European intensive care units: an epidemiological survey of the GenOSept cohort. Crit Care. 2014;18(2):R58.

10. Gaillat J, Flahault A, deBarbeyrac B, Orfila J, Portier H, Ducroix J-P, et al. Community epidemiology of Chlamydia and Mycoplasma pneumoniae in LRTI in France over 29 months. Eur J Epidemiol. 2005;20(7):643-51.

11. Sopena N, Sabrià M, Pedro-Botet ML, Manterola JM, Matas L, Domínguez $J$, et al. Prospective study of community-acquired pneumonia of bacterial etiology in adults. Eur J Clin Microbiol Infect Dis. 1999:18(12):852-8.

12. Khoury T, Sviri S, Rmeileh AA, Nubani A, Abutbul A, Hoss S, et al. Increased rates of intensive care unit admission in patients with Mycoplasma pneumoniae: a retrospective study. Clin Microbiol Infect. 2016;22(8):711-4.

13. Cillóniz C, Ewig S, Ferrer M, Polverino E, Gabarrús A, de la Bellacasa JP, et al. Community-acquired polymicrobial pneumonia in the intensive care unit: aetiology and prognosis. Crit Care. 2011;15(5):R209.

14. Lui G, Ip M, Lee N, Rainer TH, Man SY, Cockram CS, et al. Role of'atypical pathogens' among adult hospitalized patients with community-acquired pneumonia. Respirol Carlton Vic. 2009;14(8):1098-105.

15. Miyashita N, Obase Y, Ouchi K, Kawasaki K, Kawai Y, Kobashi Y, et al. Clinical features of severe Mycoplasma pneumoniae pneumonia in adults admitted to an intensive care unit. J Med Microbiol. 2007;56(Pt 12):1625-9.

16. Chan ED, Welsh CH. Fulminant Mycoplasma pneumoniae pneumonia. West J Med. 1995;162(2):133-42.

17. Yin Y-D, Zhao F, Ren L-L, Song S-F, Liu Y-M, Zhang J-Z, et al. Evaluation of the Japanese Respiratory Society guidelines for the identification of Mycoplasma pneumoniae pneumonia. Respirol Carlton Vic 2012;17(7):1131-6.

18. Guo Q, Li H-Y, Zhou Y-P, Li M, Chen X-K, Peng H-L, et al. Associations of radiological features in Mycoplasma pneumoniae pneumonia. Arch Med Sci AMS. 2014:10(4):725-32

19. Daxboeck F, Krause R, Wenisch C. Laboratory diagnosis of Mycoplasma pneumoniae infection. Clin Microbiol Infect. 2003;9(4):263-73.

20. Le Gall JR, Lemeshow S, Saulnier F. A new Simplified Acute Physiology Score (SAPS II) based on a European/North American multicenter study. JAMA. 1993;270(24):2957-63.

21. Waites KB, Talkington DF. Mycoplasma pneumoniae and its role as a human pathogen. Clin Microbiol Rev. 2004;17(4):697-728.

22. Norisue Y, Tokuda Y, Koizumi M, Kishaba T, Miyagi S. Phasic characteristics of inspiratory crackles of bacterial and atypical pneumonia. Postgrad Med J. 2008;84(994):432-6

23. Ruiz-González A, Falguera M, Vives M, Nogués A, Porcel JM, Rubio-Caballero M. Community-acquired pneumonia: development of a bedside predictive model and scoring system to identify the aetiology. Respir Med. 2000;94(5):505-10

24. Menéndez R, Torres A, Reyes S, Zalacain R, Capelastegui A, Aspa J, et al. Initial management of pneumonia and sepsis: factors associated with improved outcome. Eur Respir J. 2012;39(1):156-62.

25. Medjo B, Atanaskovic-Markovic M, Radic S, Nikolic D, Lukac M, Djukic S. Mycoplasma pneumoniae as a causative agent of community-acquired pneumonia in children: clinical features and laboratory diagnosis. Ital J Pediatr. 2014;18(40):104. 